\title{
Double-blind, randomized trial comparing efficacy and safety of continuing olanzapine versus switching to quetiapine in overweight or obese patients with schizophrenia or schizoaffective disorder
}

\author{
Walter Deberdt' \\ Ilya Lipkovich ${ }^{2}$ \\ Alexandra $\mathrm{N}$ Heinloth ${ }^{3}$ \\ Lin Liu $^{2}$ \\ Sara Kollack-Walker ${ }^{2}$ \\ Sara E Edwards ${ }^{2}$ \\ Vicki Poole Hoffmann ${ }^{2}$ \\ Thomas A Hardy² \\ 'Eli Lilly Benelux, Eli Lilly and \\ Company, Brussels, Belgium; ${ }^{2}$ Lilly \\ Research Laboratories, Eli Lilly and \\ Company, Indianapolis, IN, USA; \\ ${ }^{3}$ i3Statprobe, Ingenix, Cary, NC, USA
}

Correspondence:Walter Deberdt 52 Stoofstraat, B - 1000 Brussels, Belgium Tel +3225488615

$\mathrm{Fax}+3225488485$

Email deberdt_walter@lilly.com

\begin{abstract}
We examined the potential risks and benefits of switching from olanzapine to quetiapine in mentally stable, obese, or overweight patients with schizophrenia or schizoaffective disorder. Patients receiving olanzapine were randomized to continuing olanzapine treatment $(\mathrm{N}=68 ; 7.5-20 \mathrm{mg} /$ day $)$ or switching to quetiapine $(\mathrm{N}=65 ; 300-800 \mathrm{mg} /$ day $)$. Time to relapse was the primary study objective; secondary objectives included changes in weight, metabolic parameters, and psychiatric symptoms, and discontinuation rates. No significant difference in time to relapse was observed $(\mathrm{p}=0.293)$, but significantly more patients remained on treatment in the olanzapine group compared with the quetiapine group (70.6\% vs $43.1 \%$; $\mathrm{p}=0.002$ ). Olanzapine-treated patients had significantly lower rates of study discontinuation for lack of efficacy and psychiatric adverse events (AEs) compared to quetiapine $(2.94 \% \mathrm{vs}$ $15.38 \%, \mathrm{p}=0.015)$. Significantly more patients in the olanzapine group experienced an increase in BMI $\geq 1 \mathrm{~kg} / \mathrm{m}^{2}$. Olanzapine-treated patients experienced significantly greater increases in weight from Weeks 2 through 13. Switching patients with stable disease from olanzapine to quetiapine did not significantly shorten time to relapse, but produced more frequent study discontinuations due to lack of efficacy or psychiatric AEs with moderate but variable improvement in weight and no significant between-group differences in mean changes in metabolic laboratory parameters.
\end{abstract}

Keywords: antipsychotic switching, discontinuation, relapse, BMI, weight

\section{Introduction}

Continuous treatment with antipsychotic medication is essential for stabilization and prevention of relapse in patients with schizophrenia. Poor patient compliance undermines therapeutic success and increases the risk of hospitalization (Weiden et al 2004). Unfortunately, only about $50 \%$ of all patients receiving treatment for schizophrenia adhere to their prescribed medication regimens (Cramer and Rosenheck 1998).

Currently olanzapine, a second-generation, atypical antipsychotic drug, is one of the commonly used medications for the treatment of schizophrenia. Compared with other conventional and atypical antipsychotics, olanzapine has been associated with longer time to discontinuation due to any cause (Ascher-Svanum et al 2006; Essock et al 2006; Beasley et al 2007), which is an index thought to reflect both efficacy and tolerability of an antipsychotic medication. However, clinically significant weight gain has been frequently reported during olanzapine treatment (Kinon et al 2005). To avoid the potential consequences of clinically significant weight gain, some clinicians advocate proactive switching of stable patients from olanzapine to quetiapine, 
as this strategy has been associated with reductions in weight (Gupta et al 2004).

Switching antipsychotic medications may be one approach to lessen potential safety concerns in patients who have experienced treatment-emergent weight gain, but the impact of switching on psychiatric symptoms is less clear. The purpose of this study was to gain better understanding of potential risks and benefits associated with switching from olanzapine to quetiapine in stable patients with schizophrenia or schizoaffective disorder.

\section{Materials and methods}

This study was conducted in 26 centers in the US. The study protocol was reviewed and approved by individual institutional review boards prior to enrolling any patients, and was conducted in conformity with the US Food and Drug Administration Code of Federal Regulations (21 CFR, Part 50), and the Declaration of Helsinki and its amendments. The study was consistent with Good Clinical Practices and all applicable regulatory requirements. All participants provided written informed consent before receiving any study therapy or undergoing any study procedure. The study was conducted between July 2004 and March 2006.

\section{Patients}

Males and females between 18 and 75 years of age and diagnosed with schizophrenia or schizoaffective disorder according to the Diagnostic and Statistical Manual of Mental Disorders, Fourth Edition (DSM-IV) were enrolled in this study. Important inclusion criteria included: a confirmed psychotic episode within the last 5 years prior to enrollment; clinically stable for at least 15 days on a fixed dose of olanzapine (10-20 mg/day) prior to enrollment; obese (body mass index $[\mathrm{BMI}] \geq 30 \mathrm{~kg} / \mathrm{m}^{2}$ ) or overweight (BMI $\geq 25 \mathrm{~kg} / \mathrm{m}^{2}$ and $<30 \mathrm{~kg} / \mathrm{m}^{2}$ ) with at least one cardiovascular risk factor (diabetes mellitus or impaired fasting glucose, dyslipidemia, elevated blood pressure, or waist circumference $>102 \mathrm{~cm}$ for men or $>88 \mathrm{~cm}$ for women). Patients were to be free of any other significant medical illness at enrollment. In general, concomitant medications with primary central nervous system activity were not allowed in this protocol.

\section{Study design}

This multicenter trial utilized a 2-phase, randomized, double-blind, flexible-dosed, parallel-group design. Eligible and consenting patients were randomly assigned to receive olanzapine $(7.5-20 \mathrm{mg} /$ day) or quetiapine
(300-800 $\mathrm{mg} /$ day). During the first (double-blind, cross-titration) phase, patients randomized to the olanzapine group maintained their original dose while patients randomized to the quetiapine group had their olanzapine dose gradually decreased (and completely discontinued by Day 7) as their quetiapine dose was gradually increased. During the second (double-blind, flexible-dose) phase, doses could be adjusted within a range from $7.5 \mathrm{mg} /$ day to $20 \mathrm{mg}$ /day for olanzapine or $300 \mathrm{mg} /$ day to $800 \mathrm{mg} /$ day for quetiapine depending on patient symptoms and tolerance. Treatment was to continue for 24 weeks, followed by a 1 -week titration phase to open-label treatment at the end of the study. Over the 26 weeks total length of the study, 14 visits were scheduled for each patient; 12 of those visits occurred during the blinded study period with increasing time intervals between visits (3 visits 1 week apart, 2 visits 2 weeks apart, and 7 visits 3 weeks apart).

\section{Efficacy and safety assessments}

The primary study objective was time to relapse based on change in Positive and Negative Syndrome Scale (PANSS) (Kay et al 1987), the Clinical Global Impression of Severity (CGI-S) (Guy 1976) scale, or the level of psychiatric care. Relapse was defined as the occurrence of at least 1 of the following 3 events: hospitalization due to psychiatric reasons; $\geq 20 \%$ worsening on the PANSS total score and an increase in the level of care for psychiatric reasons compared to baseline; $\geq 20 \%$ worsening of PANSS total score and worsening of CGI-S by at least 1 level compared with baseline and CGI-S score of $\geq 4$. Secondary objectives included assessment of time to discontinuation, changes in weight, BMI, and metabolic parameters, and the incidence of treatment-emergent adverse events. The protocol specified to group discontinuations due to lack of efficacy and adverse psychiatric events together in the analysis, as treatment-emergent psychiatric adverse events are in the majority of the cases based on efficacy problems and not on tolerance problems (Liu-Seifert et al 2005; Perkins et al 2007). PANSS and CGI-S were administered at every visit.

Safety evaluations included the assessment of vital signs and weight, recording of adverse events, and review of treatment compliance at every visit. A physical examination, measurement of height and waist circumference, and an extensive interview to capture the patient's psychiatric and medical history were performed at the first visit. Laboratory tests (clinical chemistry, hematology, hepatitis, drug screen, pregnancy, TSH, prolactin, hemoglobin A1c [HbA1c], lipid panel, and insulin) 
were performed at Visit 1, and selected laboratory tests (clinical chemistry, hematology, prolactin, HbA1c, lipid panel, and insulin) were repeated throughout the study.

\section{Statistical analysis}

In the original power calculations, a sample size of approximately 200 patients per treatment group was established in order to detect a difference of $15 \%$ in rates of relapse by Week 24 with a power of $80 \%-85 \%$ and $5 \%$ type I error for a 2 -sided log rank test. Due to recruitment difficulties, only 133 patients (olanzapine, 68; quetiapine, 65) were enrolled, which yielded a reduced power of $37 \%-40 \%$ to detect a $15 \%$ difference using the same assumptions. The final sample size provided $80 \%$ power to detect a $1.0 \mathrm{~kg} / \mathrm{m}^{2}$ difference in BMI ( $29 \%$ for a $0.5 \mathrm{~kg} / \mathrm{m}^{2}$ difference) and $99 \%$ power to detect a treatment difference of $0.4 \%$ change in HbA1c.

The primary study objective was assessed using a log-rank test for differences over time in relapse for olanzapine vs quetiapine. Rates of study discontinuation by treatment group were compared using Fisher's exact test. Both the distribution of time to relapse and the survival distributions for time to discontinuation were evaluated within each group using the Kaplan-Meier product limit estimator. Treatment differences in the survival distribution were assessed using the log-rank test.

Treatment differences in categorical safety variables (occurrence of treatment-emergent adverse events, vital signs, rates of study discontinuation) were evaluated with Fisher's exact test. Analysis of variance (ANOVA) or analysis of covariance (ANCOVA) methods were used on rank changes for laboratory parameters (or raw changes for weight and BMI) from baseline to last observation carried forward (LOCF) endpoints, with terms for categorical effects of treatment and center in the model, and also adjusted for differences in baseline levels (included as a continuous score for weight and BMI or as a class variable, based on quintiles of baseline score, for laboratory parameters). Significance of changes from baseline in laboratory parameters and weight were evaluated with a nonparametric Wilcoxon signed-rank test. The cut-offs for clinically significant changes in weight and BMI were determined a priori in the study protocol.

Time course and treatment differences for changes in weight, BMI, and PANSS total scores were evaluated using mixed effects repeated measures analysis (MMRM) with categorical effects of treatment, investigator, duration of treatment, treatment by time interaction and continuous baseline score, and baseline score by time interaction. Correlation in repeated measures was modeled with unstructured covariance.
To evaluate the potential effect of differences in certain baseline covariates (BMI and PANSS) on treatment differences in categorical safety variables (categorical changes in BMI and weight, and study discontinuation), additional analyses were conducted using a "stratified version" of Fisher's exact test. Specifically, we used an exact permutation test for comparing a categorical outcome across 2 treatment groups, stratified by baseline covariate (BMI or PANSS, depending on the outcome). Strata were defined by quintiles of the baseline covariate.

Efficacy and safety analyses included all randomized patients who had at least 1 post-baseline evaluation for a given analysis. All tests of treatment effects were performed at a 2-sided alpha level of 0.05, and no adjustments for multiplicity were made. All statistical computations were performed using SAS (SAS ${ }^{\circledR}$ ) software Version 8.2 (SAS Institute, Inc., Cary, NC, USA).

\section{Results}

\section{Patients}

A total of 133 patients were randomly assigned to continuing therapy with olanzapine $(\mathrm{N}=68$; mean modal dose: $16.9 \mathrm{mg} /$ day) or a switching of treatment to quetiapine $(\mathrm{N}=65$; mean modal dose: $439.7 \mathrm{mg} /$ day). Baseline patient characteristics were similar for both treatment groups (eg, olanzapine vs quetiapine, respectively: mean age (years) [standard deviation (SD)]: 45.4 [9.4] vs 42.5 [11.5]; $\mathrm{p}=0.083$; mean time on olanzapine (weeks) [SD]: 67.5 [98.5] vs 69.4 [107.8]; $p=0.554$ ). At baseline, there were statistically significant differences between treatment groups (olanzapine vs quetiapine, respectively) in PANSS total (mean value [SD]): 61.1 [17.9] vs 65.9 [20.4]; $\mathrm{p}=0.033$ and BMI (mean value [SD]): $34.6 \mathrm{~kg} / \mathrm{m}^{2}$ [7.1] vs $37.5 \mathrm{~kg} / \mathrm{m}^{2}$ [8.6]; $\mathrm{p}=0.042$ ). These differences had occurred by chance in the random allocation process.

A significantly higher proportion of patients in the olanzapine group completed the 24 weeks of treatment compared to the quetiapine group $(70.6 \%$ vs $43.1 \%$, respectively; $p=0.002$ ). Survival curves demonstrating the probability of remaining on assigned treatment are shown in Figure 1a.

\section{Efficacy endpoints}

No significant differences were observed between the treatment groups in time to relapse (Figure 2) or in the frequency of relapses occurring during the study. In the olanzapine group, 8 patients met at least 1 of the relapse criteria compared with 10 patients in the quetiapine group (Table 1). 


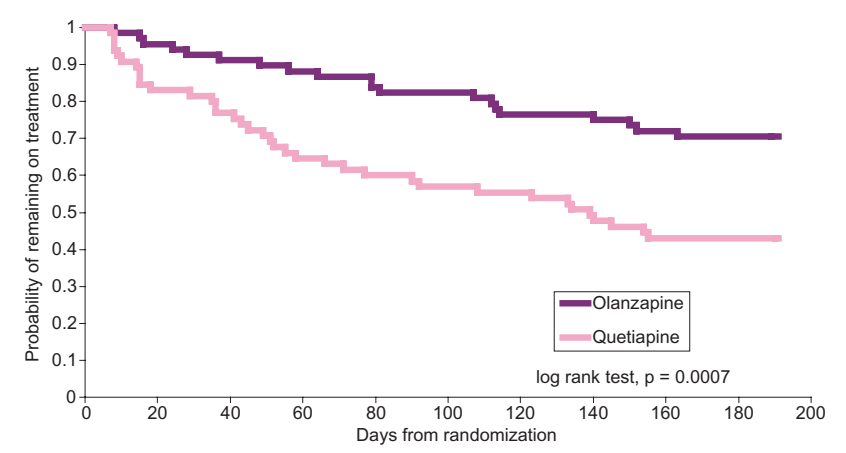

\begin{tabular}{|c|c|c|c|c|c|c|c|c|c|c|c|}
\hline Visit & $\mathbf{3}$ & $\mathbf{4}$ & $\mathbf{5}$ & $\mathbf{6}$ & $\mathbf{7}$ & $\mathbf{8}$ & $\mathbf{9}$ & $\mathbf{1 0}$ & $\mathbf{1 1}$ & $\mathbf{1 2}$ & $\mathbf{1 3}$ \\
\hline Olanzapine & 68 & 66 & 64 & 63 & 62 & 60 & 58 & 55 & 52 & 50 & 48 \\
\hline Quetiapine & 65 & 59 & 55 & 54 & 48 & 42 & 39 & 36 & 35 & 32 & 28 \\
\hline
\end{tabular}

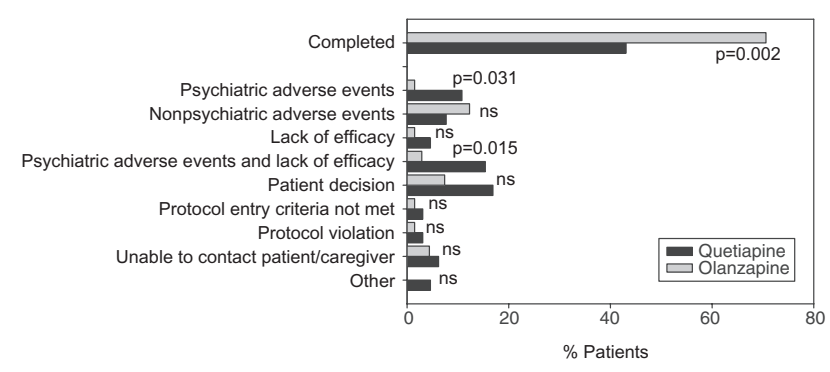

Figure I Patient disposition.

Figure Ia (upper) Time remaining on treatment. Kaplan-Meier curves estimating the probability of remaining in the study for patients treated with olanzapine or quetiapine.

Figure Ib (lower) Reasons for discontinuation. "Other” includes discontinuations due to patient incarceration.

Analysis of the reported reasons for discontinuation revealed that discontinuation due to psychiatric adverse events, or due to the combination of lack of efficacy or psychiatric adverse events, was significantly higher in the quetiapine group than in those remaining on olanzapine $(p=0.031$ and 0.015 , respectively; Figure $1 b)$. No significant difference was observed in the frequency of discontinuations due to nonpsychiatric adverse events.

Both patients treated with olanzapine and those switched to quetiapine experienced improvements in their PANSS total scores throughout the course of the study (Figure 3). While PANSS scores for patients receiving olanzapine significantly improved from baseline scores at Week 1, the improvement for quetiapine patients reached significance at Week 2. The olanzapine group maintained a slight but steady increase in improvement through the course of the blinded treatment phase. In comparison, the quetiapine group experienced less of an improvement which was not persistent throughout the blinded treatment phase. At Weeks 13 and 19, improvement from baseline was no longer significant for patients in the quetiapine group, and their PANSS total scores were significantly worse than those of patients in the olanzapine group.

\section{Changes in weight, BMI, vital signs, and metabolic laboratory parameters}

MMRM analysis of the mean changes from baseline to endpoint revealed significant increases $(p<0.05)$ for weight from Week 1 through Week 16 for patients in the olanzapine group (Figure 4a). Weight changes were statistically significant between treatment groups from Weeks 2 through Week $13(\mathrm{p}<0.05)$, with patients in the olanzapine group gaining more weight than patients in the quetiapine group (Figure 4a). No significant changes in weight were observed for patients in the quetiapine group throughout the study or for patients in the olanzapine group at the last scheduled visit. The between group differences in both categorical weight loss and weight gain (measured as a change of $\geq 7 \%$ from the baseline body weight) did not reach statistical significance.

MMRM analysis of the mean changes from baseline to endpoint revealed significant increases $(p<0.05)$ for BMI from Week 1 through Week 13 in the olanzapine group (Figure 4b). Significantly more patients in the olanzapine group than in the quetiapine group experienced an increase in BMI $\geq 1 \mathrm{~kg} / \mathrm{m}^{2}$ during treatment with olanzapine compared with quetiapine (53.7\% vs $33.3 \%, \mathrm{p}=0.031)$. No significant between-group difference was observed in the number of patients whose BMI decreased $\geq 1 \mathrm{~kg} / \mathrm{m}^{2}$ during treatment with olanzapine compared with quetiapine $(30.9 \%$ vs $39.3 \%$, $\mathrm{p}=0.357)$.

Vital signs showed no significant between group differences. Changes in HbAlc were small in both groups and the difference was not statistically significant (olanzapine $+0.07 \%$, quetiapine $-0.03 \%$ ). LOCF analysis of the mean changes from baseline to LOCF endpoint for weight, BMI, fasting glucose, insulin, triglycerides, HDL-cholesterol, LDL-cholesterol, or total cholesterol did not demonstrate significant differences between treatment groups (Table 2); olanzapine vs quetiapine: weight at baseline $(\mathrm{kg})$ [SD]: 100.71 [19.7] vs 106.7 [19.9]; mean change in weight to LOCF endpoint ( $\mathrm{kg}$ ) [SD]: 0.99 [5.82] vs -0.82 [5.30], $\mathrm{p}=0.088$; BMI at baseline $\left(\mathrm{kg} / \mathrm{m}^{2}\right)$ [SD]: 34.6 [7.1] vs 37.5 [8.6]; mean change in BMI to LOCF endpoint $\left(\mathrm{kg} / \mathrm{m}^{2}\right)$ [SD]: 0.31 [1.94] vs -0.30 [1.90], $p=0.150$ ). Fasting insulin levels were elevated at baseline and did not improve in either group. 


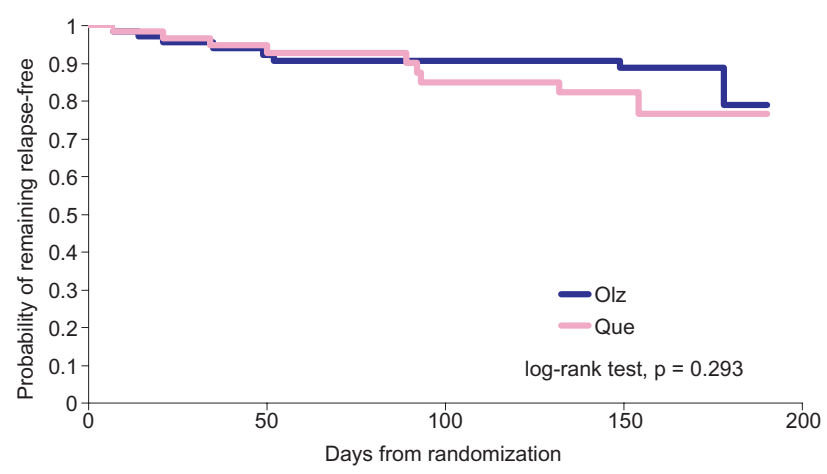

Figure 2 Time remaining relapse free. Kaplan-Meier curves estimating the probability of remaining relapse free for patients treated with olanzapine or quetiapine.

\section{Other safety evaluations}

\section{Treatment-emergent adverse events (TEAEs)}

No statistically significant difference was observed for TEAEs between treatment groups. The most common TEAEs $(\geq 5 \%)$ in the olanzapine treatment group were sedation, vomiting, anxiety, hypertension, insomnia, pharyngolaryngeal pain, somnolence, weight decrease, and weight increase. In the quetiapine treatment group, the most common TEAEs $(\geq 5 \%)$ were sedation, anxiety, insomnia, weight increase, headache, constipation, dry mouth, auditory hallucination, paranoia, and agitation.

\section{Discussion}

In this randomized, double-blind, flexible-dosed trial, we examined the potential risks and benefits of switching from olanzapine to quetiapine mentally stable obese or overweight patients with schizophrenia or schizoaffective disorder. No significant difference was seen in the time to or incidence of psychiatric relapse using this strategy. However, significant between-group differences were observed in the rates of study discontinuation due to any reason, due to psychiatric adverse events, or due to a combination of lack of efficacy and psychiatric AEs (all three were higher in the quetiapine group), and the rates of categorical BMI increase (higher in the olanzapine group). No statistically significant between-group difference was observed in the rates of study discontinuation due to nonpsychiatric adverse events. Mean changes in weight, BMI, fasting glucose, insulin, $\mathrm{HbA1c}$, and lipids from baseline to endpoint by LOCF method were generally small and did not differ significantly between groups.

This study illustrates the importance of evaluating both efficacy and safety when examining the potential effects of treatment changes on mentally stable patients. While most studies examining the potential effects of switching patients from olanzapine to another antipsychotic focus on metabolic parameters, they do not always report potential changes in psychiatric symptoms (Garman et al 2007). Study discontinuation due to any reason is thought to serve as a measure of efficacy and safety (Essock et al 2006). In this analysis, we found significant differences in study discontinuation rates in favor of olanzapine, which may reflect this composite measure.

The increased rate of study discontinuation in the quetiapine group due to lack of efficacy and occurrence of psychiatric events may indicate a prodromal worsening in symptoms that led to study discontinuation prior to relapse (as defined in this study). Overall, this finding suggests that switching a stable patient from olanzapine to another antipsychotic medication may present additional risk for treatment discontinuation, a finding that has been previously reported (Essock et al 2006). Alternatively, the fact that the quetiapine treatment group had a significantly higher discontinuation rate - resulting in a shorter duration of drug exposure - may have affected the analyses of relapse rate and time to relapse. Limiting the interpretation is the possibility that some of the patients that discontinued early in the course of quetiapine

Table I Relapse rate by treatment group

\begin{tabular}{|c|c|c|c|c|}
\hline Relapse criteria & Olanzapine $(\mathrm{N}=68)$ & Quetiapine $(N=65)$ & Total $(\mathbf{N}=133)$ & p value ${ }^{a}$ \\
\hline $\begin{array}{l}\text { Hospitalization for psychiatric } \\
\text { reasons after Visit } 2\end{array}$ & I (I.47\%) & $5(7.69 \%)$ & $6(4.51 \%)$ & ns \\
\hline $\begin{array}{l}\geq 20 \% \text { worsening in PANSS Total score } \\
\text { and increase in Level of Care for psychiatric } \\
\text { reason after Visit } 2\end{array}$ & 0 & $2(3.08 \%)$ & $2(1.5 \%)$ & ns \\
\hline $\begin{array}{l}\geq 20 \% \text { worsening on the PANSS Total score } \\
\text { and worsening of CGI-S by at least one level } \\
\text { compared to baseline and CGI-S score } \geq 4\end{array}$ & 7 (10.29\%) & 7 (10.77\%) & 14 (10.5\%) & ns \\
\hline Patients meeting at least one of the above criteria & $8(11.76 \%)$ & $10(15.38 \%)$ & $18(13.5 \%)$ & ns \\
\hline
\end{tabular}

${ }^{a} \mathrm{P}$ value is based on Fisher's exact test for treatment difference.

Abbreviations: CGI-S, Clinical Global Impression of Severity; ns, not significant; PANSS, Positive and Negative Syndrome Scale. 


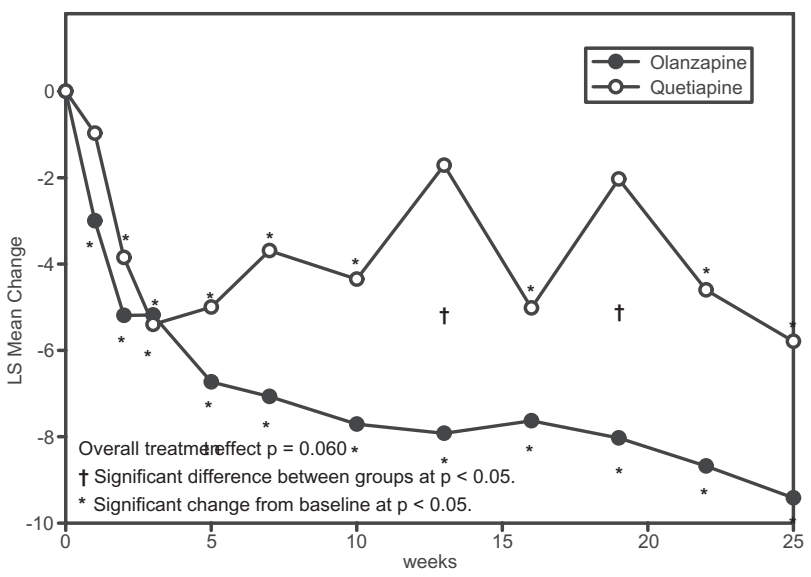

Figure 3 Repeated measures analysis of changes in PANSS Total Score. Least-squares means (LSMEANS) for changes in PANSS from baseline to each scheduled visit estimated with a MMRM model that included terms for investigator, drug, PANSS baseline score, visit-by-baseline score and visit-by-drug interactions.

treatment might have done so due to eventual withdrawal symptoms potentially related to their prior treatment with olanzapine.

Our results are supported by the outcome of the CATIE study. There, patients who had discontinued treatment with the atypical antipsychotic they had been assigned to in phase I were randomly reassigned to olanzapine, risperidone, quetiapine, or ziprasidone. Direct comparison of time to discontinuation between all 4 groups revealed superior performance of olanzapine and risperidone over quetiapine and ziprasidone (Stroup et al 2006).

Our analysis did not detect significant between-group differences in treatment-emergent changes in metabolic laboratory parameters, which is contrary to published reports that have reported serum lipid level decreases after switching from olanzapine to other antipsychotics (Montes et al 2007). Patients in this trial may have differed from those in previous studies in terms of severity and duration of illness, or the higher rate of study discontinuation in the quetiapine group may have limited the ability to detect significant differences in LOCF analysis of changes in weight, BMI, and other metabolic parameters. The MMRM method, known to be less affected by patient dropouts (Mallinckrodt et al 2003), showed a significantly greater increase in weight and BMI occurring in patients who continued receiving olanzapine than in those who switched to quetiapine. While the overall MMRM analysis revealed statistically significant $\mathrm{p}$ values for weight $(p=0.020)$ and BMI $(p=0.035)$, the difference at last scheduled visit using this method was not significant. For the difference in rate of BMI increase, we have to realize that the exposure time in the olanzapine group is much higher, which makes the interpretation of this result very difficult. Despite adequate power to detect significant differences in several metabolic parameters, conclusions regarding changes in the potential risk of treatment-emergent changes in metabolic parameters after switching from olanzapine to quetiapine should be viewed with caution based on the data presented here. Nonetheless, the differences in weight were generally small, which is a finding similar to that reported by Gupta et al who observed a change in mean BMI from $35.1 \mathrm{~kg} / \mathrm{m}^{2}$ to $34.4 \mathrm{~kg} / \mathrm{m}^{2}(\mathrm{p}=0.065)$ and a change in mean weight from $103 \mathrm{~kg}$ to $101 \mathrm{~kg}(\mathrm{p}=0.03)$ in an open-label study in which 16 patients were switched from olanzapine to quetiapine. In that study, only 12 patients completed treatment and 1 patient discontinued due to exacerbation of psychosis (Gupta et al 2004). Comparable changes in weight and BMI have been observed after the addition of metformin (Baptista et al 2007) or amantadine (Deberdt et al 2005) to olanzapine treatment as weight-mitigating agents, without increasing the risk for discontinuation due to increased psychiatric symptoms.

Table 2 LOCF analysis of weight, BMI, and metabolic laboratory parameters

\begin{tabular}{|c|c|c|c|c|c|c|c|}
\hline \multirow[t]{2}{*}{ Parameter } & \multicolumn{3}{|c|}{ Olanzapine $(n=68)$} & \multicolumn{4}{|c|}{ Quetiapine $(n=65)$} \\
\hline & $\begin{array}{l}\text { Baseline } \\
\text { (SD) }\end{array}$ & $\begin{array}{l}\text { Change } \\
\text { (SD) }\end{array}$ & $p$ value & $\begin{array}{l}\text { Baseline } \\
\text { (SD) }\end{array}$ & $\begin{array}{l}\text { Change } \\
\text { (SD) }\end{array}$ & $p$ value & $\begin{array}{l}\text { Between-group } \\
\text { p value }\end{array}$ \\
\hline Weight (kg) & I00.7I (19.73) & $0.99(5.82)$ & 0.157 & $106.74(19.87)$ & $-0.82(5.30)$ & 0.204 & 0.088 \\
\hline BMI & $34.55(7.14)$ & $0.31(1.94)$ & 0.153 & $37.45(8.57)$ & $-0.30(1.90)$ & 0.205 & 0.150 \\
\hline Fasting glucose $(\mathrm{mmol} / \mathrm{L})$ & $5.58(1.56)$ & $-0.01(1.53)$ & 0.622 & $5.36(1.46)$ & $-0.06(1.08)$ & 0.693 & 0.228 \\
\hline $\mathrm{HbAlc}(\%)$ & $5.82(0.84)$ & $0.07(0.48)$ & 0.292 & $5.93(0.77)$ & $-0.03(0.40)$ & 0.493 & 0.318 \\
\hline Cholsterol (mmol/L) & $5.33(1.16)$ & $-0.20(0.93)$ & 0.085 & $5.17(1.08)$ & $-0.11(0.68)$ & 0.325 & $0.47 \mathrm{I}$ \\
\hline LDL (mmol/L) & $3.36(1.00)$ & $-0.14(0.82)$ & 0.138 & $3.16(0.95)$ & $-0.07(0.5 \mathrm{I})$ & 0.243 & 0.981 \\
\hline $\mathrm{HDL}(\mathrm{mmol} / \mathrm{L})$ & I.I8 (0.3I) & $-0.03(0.21)$ & 0.487 & $1.26(0.42)$ & $-0.02(0.18)$ & 0.513 & 0.872 \\
\hline Insulin (ulU/ml) & $23.95(22.02)$ & $1.78(24.26)$ & 0.500 & $30.49(46.67)$ & $3.62(74.72)$ & 0.894 & 0.262 \\
\hline Triglycerides (mmol/L) & $2.1(1.23)$ & $-0.11(0.98)$ & 0.314 & $1.96(1.34)$ & $0.07(0.90)$ & 0.379 & 0.167 \\
\hline
\end{tabular}

Abbreviations: BMI, body mass index; LOCF, last observation carried forward; SD, standard deviation. 
The study was designed to allow optimal use of each antipsychotic. The mean modal dose of quetiapine (439.7 mg/day) used during the flexible dosing phase was consistent with current dosing recommendations for quetiapine (target dose range for schizophrenia: $300 \mathrm{mg} /$ day to $450 \mathrm{mg}$ /day according to prescribing information at http:// www.seroquel.info/). All patients had reached the target dose range at the end of the cross-titration period, and quetiapine dosing up to $800 \mathrm{mg}$ /day was allowed depending on patient symptoms and the investigator's discretion. While the first study discontinuations occurred after the cross-titration phase, the treatment survival curves continued to diverge well
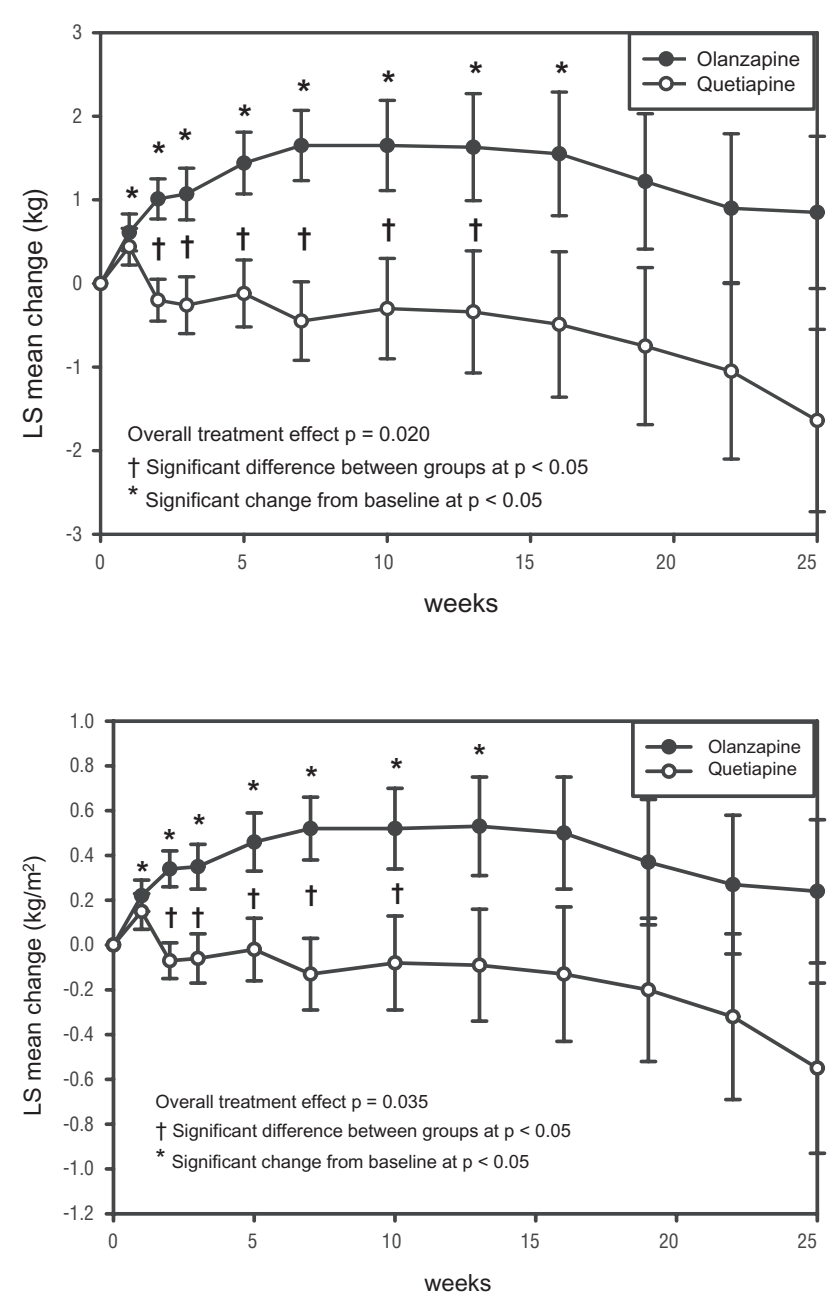

Figure 4 Changes in $\mathrm{BMI}$ and weight.

Figure $4 \mathrm{a}$ (upper) Repeated measures analysis of weight changes. Least-squares means (LSMEANS) for changes in weight $(\mathrm{kg})$ from baseline to each scheduled visit estimated with MMRM model that includes terms for investigator, drug, baseline weight, visit-by-baseline score and visit-by-drug interactions. The vertical error bars indicate LSMEANS standard error.

Figure $\mathbf{4 b}$ (lower) Repeated measures analysis of changes in BMI. Least-squares means (LSMEANS) for changes in BMI $\left(\mathrm{kg} / \mathrm{m}^{2}\right)$ from baseline to each scheduled visit estimated with MMRM model that included terms for investigator, drug, baseline BMI, visit-by-baseline score, and visit-by-drug interactions. The vertical error bars indicate LSMEANS standard error. beyond this period; thus it is unlikely that the cross-titration regimen explains the higher rate of study discontinuations in the quetiapine group.

This study had several limitations. The most significant was reduced sample size in both treatment groups. The study was terminated early due to slow enrollment, although the decision to terminate was not based on an interim analysis of un-blinded data, which ensures that subsequent analyses had no inherent selection bias. Nevertheless, termination before the recruitment goal was reached diminishes the power of the statistical analysis and substantially reduces the probability to detect a possible treatment difference in relapse or other measures of efficacy and safety.

Further limitations were the significant between-group differences seen at baseline for PANSS total score and BMI. These could have affected testing for treatment effect for categorical variables using Fisher's exact test at endpoints that were not adjusted for these baseline differences. To reduce possible bias due to these imbalances, we reanalyzed categorical changes in weight and BMI (stratified by baseline BMI), as well as study discontinuation due to psychiatric reasons and lack of efficacy (stratified by baseline PANSS total score) using a stratified version of Fisher's exact test. These reanalyzes produced very similar results with the same significance levels as the unadjusted analysis.

In conclusion, we were able to demonstrate that switching from olanzapine to quetiapine in mentally stable patients can increase rate of study discontinuations in particular because of emergent psychiatric symptoms, although significantly fewer quetiapine-treated patients experienced an increase in BMI and some modest weight differences in favor of the quetiapine arm were observed. This suggests that in general, patients remaining on olanzapine therapy are more likely to stay stable and to continue treatment compared with those switched to quetiapine. Therefore, treating clinicians should carefully evaluate the potential risk for treatment discontinuation due to symptom exacerbation when considering a switch from one antipsychotic to another in psychiatrically stable patients.

\section{Acknowledgments}

This work was sponsored by Eli Lilly and Company. Drs. Deberdt, Lipkovich, Poole Hoffmann, Edwards, Liu, KollackWalker and Hardy are employees of Eli Lilly and Company. Dr. Heinloth is a scientific writer employed full-time by i3 Statprobe, a division of Ingenix, which is a subsidiary of UnitedHealth Group. Eli Lilly contracted the technical writing of this manuscript with i3 Statprobe. The authors thank Caron Modeas for editorial assistance. 


\section{References}

Ascher-Svanum H, Zhu B, Faries D, et al. 2006. Time to discontinuation of atypical versus typical antipsychotics in the naturalistic treatment of schizophrenia. BMC Psychiatry, 6:8.

Baptista T, Rangel N, Fernandez V, et al. 2007. Metformin as an adjunctive treatment to control body weight and metabolic dysfunction during olanzapine administration: A multicentric, double-blind, placebocontrolled trial. Schizophr Res, 93:99-108.

Beasley CM Jr, Stauffer VL, Liu-Seifert H, et al. 2007. All-cause treatment discontinuation in schizophrenia during treatment with olanzapine relative to other antipsychotics: an integrated analysis. J Clin Psychopharmacol, 27:252-8.

Cramer JA, Rosenheck R. 1998. Compliance with medication regimens for mental and physical disorders. Psychiatr Serv, 49:196-201

Deberdt W, Winokur A, Cavazzoni PA, et al. 2005. Amantadine for weight gain associated with olanzapine treatment. Eur Neuropsychopharmacol, $15: 13-21$.

Essock SM, Covell NH, Davis SM, et al. 2006. Effectiveness of switching antipsychotic medications. Am J Psychiatry, 163:2090-5.

Garman PM, Ried LD, Bengtson MA, et al. 2007. Effect on lipid profiles of switching from olanzapine to another second-generation antipsychotic agent in veterans with schizophrenia. J Am Pharm Assoc (Wash), 47:373-8.

Gupta S, Masand PS, Virk S, et al. 2004. Weight decline in patients switching from olanzapine to quetiapine. Schizophr Res, 70:57-62.

Guy W. 1976. ECDEU Assessment manual for Psychopharmacology, Revised (Publication ADM 76-338). US Dept of Health, Education, and Welfare.
Kay SR, Fiszbein A, Opler LA. 1987. The positive and negative syndrome scale (PANSS) for schizophrenia. Schizophr Bull, 13:261-76.

Kinon BJ, Kaiser CJ, Ahmed S, et al. 2005. Association between early and rapid weight gain and change in weight over one year of olanzapine therapy in patients with schizophrenia and related disorders. J Clin Psychopharmacol, 25:255-8.

Liu-Seifert H, Adams DH, Kinon BJ. 2005. Discontinuation of treatment of schizophrenic patients is driven by poor symptom response: a pooled post-hoc analysis of four atypical antipsychotic drugs. $B M C$ Med, $3: 21$.

Mallinckrodt CH, Sanger TM, Dube S, et al. 2003. Assessing and interpreting treatment effects in longitudinal clinical trials with missing data. Biol Psychiatry, 53:754-60.

Montes JM, Rodriguez JL, Balbo E, et al. 2007. Improvement in antipsychotic-related metabolic disturbances in patients with schizophrenia switched to ziprasidone. Prog Neuropsychopharmacol Biol Psychiatry, 31:383-8.

Perkins DO, Gu H, Weiden PJ, et al. 2007. Predictors of treatment discontinuation and medication nonadherence in patients recovering from a first episode of schizophrenia, schizophreniform disorder, of schizoaffective disorder: a randomized, double-blind, flexible-dose, multicenter study. J Clin Psychiatry, e1-e8 [Epub ahead of print].

Stroup TS, Lieberman JA, McEvoy JP, et al. 2006. Effectiveness of olanzapine, quetiapine, risperidone, and ziprasidone in patients with chronic schizophrenia following discontinuation of a previous atypical antipsychotic. Am J Psychiatry, 163:611-22.

Weiden PJ, Kozma C, Grogg A, et al. 2004. Partial compliance and risk of rehospitalization among California Medicaid patients with schizophrenia. Psychiatr Serv, 55:886-91. 